\title{
UNA CLASSIFICAZIONE DEI SEGNALI DISCORSIVI IN ITALIANO
}

\author{
ELIZAVETA KHACHATURYAN \\ Università di Oslo
}

\section{A B S T R ACT}

This paper presents an approach to the description of discourse markers (DMs) which is based on studies (from semantic and syntactic perspectives) of material from different languages. A classification of DMs is in the present study presented and illustrated by Italian examples. The following DMs are described : davvero, veramente, dico ; diciamo (It.) - disons (Fr.) ; tu vois (Fr.).

Each subclass (independently of the individual semantics of the word) accomplishes a particular task in the discourse and is characterised by general semantic and syntactic features. Moreover, the form of the DM can often indicate to which subclass it belongs. This kind of classification can give necessary cues for contrastive analysis, it can furthermore explain how to distinguish DMs of the same function and help in the acquisition of DMs (L2) and in translation. The advantages of this kind of approach are illustrated by several examples from Italian and French.

\section{PAROLE CHIAVE}

semantica, analisi contrastiva, comunicazione, verba dicendi

Negli anni '80 nasce l'interesse dei linguisti verso gli elementi definiti da Fraser (1990) come "extremely useful guides for clarifying a speaker's communicative intention" (1990: 390). A partire da quel momento numerose definizioni sono state proposte, diverse indagini basate su varie metodologie sono state condotte, ma ancora molte questioni legate all'uso e alla traduzione dei segnali discorsivi (SDi) rimangono aperte. Questo articolo si propone di presentare una classificazione dei SDi in italiano basata sui criteri formali e di dimostrare i vantaggi di un tale approccio.

L'articolo è organizzato nel modo seguente: all'inizio daremo una breve descrizione della metodologia che usiamo (\$1), in seguito presenteremo la classificazione dei SDi dell'italiano basata sui criteri semantico-formali (\$2), e alla fine discuteremo le applicazioni pratiche che può avere l'approccio proposto (\$3). 


\section{[1] INTRODUZIONE}

Al momento attuale nella maggior parte delle descrizioni i SDi si presentano come una classe funzionale molto eterogenea con pochi elementi comuni. Basti vedere il libro di K.Fischer "Approaches to Discourse Particles" (2006) che raccoglie diversi approcci e le metodologie più recenti. Citiamo alcune definizioni dei SDi che rappresentano bene le idee più diffuse. "Connectives are a pragmatic category, that is to say, what all connectives have in common is not the grammatical class to which they belong but their ability to signal a relationship between two units" (Pons Bordería, 2006: 82). "Discourse markers are items external to propositional content which are useful in locating the utterance in an interpersonal and interactive dimension, in connecting and structuring phrasal, inter-phrasal and extra-phrasal elements in discourse, and in marking some on-going cognitive processes and attitudes" (Bazzanella, 2006: 456).

Nella nostra analisi ci basiamo su un'approccio diverso: definiamo i SDi come classe di unità della lingua (e non le unità pragmatiche, come nelle definizioni citate sopra) con delle proprietà generali formali (e non solo funzionali). Quest'approccio è stato elaborato nell'ambito del progetto di ricerca dedicato ai SDi in russo e in francese (Università Paris 7 , coordinatore del progetto D. Paillard) ${ }^{1}$. In seguito a una descrizione sistematica di cinquanta SDi del francese e del russo (due lingue appartenenti a sistemi grammaticali diversi) sono stati formulati i criteri distribuzionali (critères distributionnels) che permettono di identificare i SDi come classe riportandoli allo stesso livello delle parti del discorso tradizionali (nomi, aggettivi, verbi, ecc.). Per lo più basandosi su queste proprietà è stata creata una classificazione dei SDi che può aiutare sia nella descrizione dei SDi in una sola lingua che nello studio contrastivo.

\section{[1.1] Proprietà generali dei segnali discorsivi}

Nell'ambito dell'approccio che presentiamo, la definizione generale del SD è basata sulla nozione di status discorsivo (statut discursif): il SD conferisce un certo status discorsivo alla sua portata (fr. portée, ing. scope) p. Questo significa che per formulare l'identità semantica del SD bisogna specificare quale stato discorsivo assume la sua portata p. "La fonction des MDs (marqueurs discursifs - E.K.) est d'introduire des déterminations supplémentaires concernant le statut du dire (noi sottolineiamo - E.K.) en tant qu'exprimant l'état de choses Z." (Paillard \& Vu Thi, in stampa)

In questa definizione bisogna specificare la nozione di dire. Il dire stesso viene definito come "une façon partiale et partielle d'exprimer par un énoncé un état

[1] Per una descrizione dettagliata v. Paillard, 1999, 2000. 
de choses du monde" (Paillard, 2009: 114)2: il modo particolare di dire $\mathbf{Z}$ ha il carattere parziale (partiel) e pregiudiziale (soggettivo) (partial). Queste due caratteristiche permanenti rispecchiano le proprietà (per non dire le difficoltà) generali della comunicazione. Si tratta del modo pregiudiziale (soggettivo) perché il mondo di cui si parla corrisponde, in realtà, solo alla visione del mondo che appartiene al locutore e quindi dipende dalle sue conoscenze, dalle sue rappresentazioni del mondo, dalla sua percezione. È caratterizzato come modo parziale perché non esiste un modo di dire assoluto, non è mai chiaro se il mondo è stato "detto" fino in fondo (jusqu'au bout). Inoltre, le parole hanno il loro proprio "vouloir dire" e possono essere interpretate in modo diverso dal locutore e dall'interlocutore. ${ }^{3}$

Così, la portata $\mathbf{p}$ del SD corrisponde a un modo particolare di dire $\mathbf{Z}$. In base alle proprietà dei SDi se ne possono individuare quattro classi: segnali discorsivi (mots du discours), particelle (particules énonciatives), modalizzatori (modalisateurs) e segnali del dire (mots du dire). Ogni classe di SDi (indipendentemente dalla semantica individuale) ha un ruolo specifico nel discorso ed i propri tratti semantico-sintattici (ne parleremo successivamente nel §3.).

Così, nella descrizione dei SDi, si parte dalle proprietà generali (di carattere semantico e morfosintattico) che descrivono tutta la classe di SDi, per arrivare poi alle proprietà che distinguono le sottoclassi, e infine, all'identità semantica di un solo SD.

\section{[1.2] Identità semantica dei segnali discorsivi}

L'identità semantica del SD rimane invariabile in qualsiasi contesto e distingue il SD da altri SDi con funzioni simili. L'identità semantica del SD viene individuata attraverso l'analisi del contesto in base agli usi possibili del SD nel contesto: definire la parola significa descrivere i contesti (nei quali essa è utilizzata) e la sua variazione nel contesto.

"Procédures et format de description sont fondés sur une hypothèse concernant l'identité des mots en tant qu'unités de la langue. Cette hypothèse postule un rapport étroit entre identité et variation du mot. L'identité est en effet indissociable de la prise en compte de la séquence (phrase ou, ce qui est le cas des MD, suite de phrases) où il apparait, et il importe dès lors, pour un mot donné, de définir les différents contextes où ce mot s'inscrit. Cela revient à dire que le mot se définit par ses usages (nés de l'interaction entre le mot et ses contextes) : l'identité du mot est dans sa variation." (Paillard 1999: 16; noi sottolineiamo - E.K.) Così, tra l'identità semantica e la variazione della parola nel contesto

[2] “(...) si le dire n'est jamais qu'un dire parmi d'autres, cela tient à ce que d'autres mots sont possibles et que rien ne garantit a priori l'adéquation des mots utilisés: d'une certaine façon, les mots échappent au locuteur car ils disent "ce qu'ils veulent dire" et rien ne dit que l'interlocuteur, dans son travail d'interprétation, interprète ce vouloir dire comme le locuteur" (Paillard, 2009: 114).

[3] Quest'assenza di un legame univoco provoca i malintesi e l'incomprensione, ma allo stesso tempo distingue la comunicazione umana dal linguaggio delle macchine (rendendo possibile, p.es. il gioco di parole e i doppi sensi). 
esiste un legame stretto: l'analisi dei contesti d'uso del SD ci porta a identificare le sue proprietà semantiche individuali. Inoltre, come vedremo successivamente, anche il lessema d'origine con il quale il SD rimane legato ha un ruolo importante nella formazione delle proprietà individuali del SD.

\section{[2] CLASSIFICAZIONE DEI SEGNALI DISCORSIVI}

La classificazione che presentiamo in questa parte è stata proposta in Paillard (2009), ed elaborata in Paillard D. \& Vu Thi N. (in stampa). Per illustrarla useremo gli esempi presi dall'italiano. Vedremo che una classificazione simile aiuta a distinguere i SDi, in particolare i SDi caratterizzati dalla stessa funzione pragmatica.

Ogni paragrafo è organizzato allo stesso modo: all'inizio definiamo le proprietà tipiche della classe (basandoci sulla descrizione proposta per i SDi delle altre lingue - Paillard, 2009 - per il russo e il francese, Paillard \& Vu Thi, in stampa per il francese e il khmer) che illustriamo dopo con gli esempi dei SDi dell'italiano.

\section{[2.1] I segnali discorsivi (classe 1)}

La classe definita come segnali discorsivi è stata studiata più delle altre classi (p.es. Kisseleva \& Paillard, 1998, Paillard, 2002). Uno dei principali problemi teorici che sorge in questo caso riguarda l'appartenenza categoriale: la maggior parte degli elementi di questa classe ha la forma degli avverbi. Per distinguere tra gli avverbi (o l'uso avverbiale) e i segnali discorsivi è stato proposto di basarsi sulla nozione di dire (Franckel \& Paillard, 2008). I SDi costituiscono un "mode de dire sur le monde", mentre gli avverbi commentano solamente il contenuto proposizionale. Così, i SDi sono definiti nel modo seguente:

"Les mots du discours (MD) ont pour fonction de spécifier à quel titre la séquence $p$, correspondant à leur portée, est une façon partiale et partielle d'exprimer un état de choses $Z$." Inoltre, due sottoclassi vengono distinte : "Selon qu'il y a prépondérance du dire ou du monde, on pourra distinguer deux sous-classes" :

- les MD adéquation (catégorisant)

- les MD points de vue (perception d'un état de choses)." (Paillard \& Vu Thi, in stampa)

Per cogliere la distinzione tra le parole di adeguatezza e le parole del punto di vista proviamo a confrontare davvero e veramente. Sono due parole a priori molto simili. Entrambe sono formate dall'aggettivo 'vero' e hanno la forma di un avverbio: vero + mente, $d a+v e r o$. Veramente è di solito definito solo come avverbio (p.es. nel dizionario di Sabatini \& Coletti), mentre davvero, nella Grande grammatica di consultazione (1994-95, capitolo sui SDi scritto da C.Bazzanella), è descritto come 
segnale discorsivo di modulazione (1995: 238). Nei dizionari di solito queste parole sono definite una attraverso l'altra (nonostante non siano interscambiabili in alcuni contesti ${ }^{4}$ ).

Possiamo notare che la semantica di davvero è basata sull'idea di adeguatezza: davvero segnala che $\mathbf{p}$ è un dire adeguato a dire $\mathbf{Z}$ che corrisponde alla verità assoluta (ciò che risale all'origine di davvero: $d a+v e r o$ ) per parlare del mondo. Veramente, invece, fa parte dei SDi punti di vista: veramente attribuisce a $\mathbf{p}$ lo status di un punto di vista possibile su Z. (Khachaturyan, 2009a)

Vediamo come queste caratteristiche si realizzano nel contesto. Confrontiamo i contesti d'uso simili: a) (p') davvero / veramente $\mathbf{p}$, dove la portata $\mathbf{p}$ del SD ha la forma di un aggettivo e spesso corrisponde a un elemento ( $p$ ') già introdotto nel contesto precedente; b) con la negazione: non è davvero / veramente $\mathbf{p}$. Vedremo come cambia lo status discorsivo dell'elemento $\mathbf{p}$ (la portata) a seconda della parola che lo introduce e come influisce sullo svolgimento del contesto.

a) (p') davvero / veramente $\mathbf{p}$

Nei due esempi riportati sotto, lo stato delle cose $\mathbf{Z}$ è caratterizzato attraverso il termine $\mathbf{p}$. Tradizionalmente si dice che davvero e veramente rafforzano il contenuto proposizionale della portata. Tuttavia, come vediamo, il contesto è organizzato in modo diverso a seconda della parola che è usata.

(1) È un film d' azione, alla Maurizio Merli, e anche un po' all' americana. Un film davvero bello, vedrete. Di solito, dei film non si racconta la fine, ma forse questa volta vale la pena sapere chi ha la meglio tra polizia e malviventi. (La Repubblica)

(2) I musicisti e i cantanti sono collocati in platea, svuotata delle poltrone. Il pubblico sta nei palchi, e sulla scena agiscono i mimi. È un modo di fare di tutto lo spazio uno spazio teatrale, lo spazio di un' azione. Bello, veramente bello. Anche perché i musicisti del Gruppo Strumentale del Teatro e / o Musica sono bravi, e così gli attori del Teatro Laboratorio Sardo Akroama, e chiari nella dizione, assai espressivi il tenore Terige Sirolli e il basso Alberto Jona, bravissimo il soprano Sonia Sigurtà. (id.)

In (1), vedrete che segue dopo la sequenza davvero bello funge quasi da conferma del termine scelto: il mondo $\mathbf{Z}$ viene categorizzato attraverso $\mathbf{p}$ (bello) - il termine adeguato per dire il mondo. In (2), il contesto posteriore spiega quali sono le proprietà che permettono di usare l'elemento $\mathrm{p}$ : tra tutte le possibilità per parlare del mondo, ne viene scelta una - bello - è un punto di vista possibile sul mondo. Questa scelta va motivata e spiegata, ciò che porta alla necessità del contesto posteriore:

[4] Per esempio nel contesto seguente: - è Leo che invita... - Veramente (*davvero) non ho invitato un corno. (Moravia) 
la spiegazione delle ragioni per le quali la definizione bello può essere usata. Negli esempi successivi si può osservare la stessa regolarità. Le definizioni grande (3) e bello (4) spiegate nel contesto posteriore (sottolineato nel testo) corrispondono alle denominazioni possibili dello stato delle cose. In (5), la sequenza davvero $\mathbf{p} \mathrm{fa}$ parte della frase finale dell'articolo: $\mathbf{p}$ non è più discusso ma semplicemente segnalato come termine adeguato che permette di categorizzare la denominazione come verità assoluta.

(3) "Vorrei, prima di finire la carriera, fare un grande film, ma veramente grande da entrare nella storia come Ladri di biciclette ". (La Repubblica)

(4) Di calcio bello, ma veramente bello ne ho visto solo una mezz' ora. Milan - Galatasaray, tre gol di Borgonovo. Per il resto non c'è stato granché. (id.)

(5) Non credo invece che siano mancati e che manchino esempi di situazioni in cui la libertà di mercato non è affatto accompagnata dalla democrazia. La democrazia ha invece un valore e un impatto che vanno ben oltre quel che comunemente si intende per libertà di mercato. Non solo non è incompatibile con un intervento consapevole nell' economia, ma è necessaria perché questo possa essere davvero efficiente. (id.)

Sulle stesse differenze è basato l'uso di davvero e veramente con la negazione.

b) non è davvero / veramente $\mathbf{p}$

Nei due esempi che seguono sia davvero che veramente sono usati con la negazione.

(6) E dire pure che, messi insieme, benché siano attenti scrittori di storie, essi non sono in grado di rinverdire o di proporre una tendenza o una indicazione, o uno stupore unitario, o una univoca ricerca di drammaturgia, non è davvero giudizio riduttivo ma è al contrario una onesta presa di contatto con la realtà, che non si modifica illudendosi di commissionarla.

(La Repubblica)

(7) Nelle due partite finali in cui Kasparov ebbe la meglio, bisogna ammettere che Karpov non era lui, ma, piuttosto, un uomo disperato, desideroso solo di farla finita. Più o meno lo stesso uomo della prima partita del match di adesso. Sì, anche adesso: Karpov che mangia in b 2 alla decima mossa e che cambia le Donne alla dodicesima in una situazione per lui così sfavorevole, non è veramente Karpov. Dopo una prima partita tanto sconcertante, la seconda assunse un aspetto del tutto particolare. (id.)

In (6), davvero con la negazione respinge l'espressione usata (giudizio riduttivo), nel contesto posteriore viene introdotta una definizione adeguata (sottolineato nel testo), l'opposizione tra $\mathbf{p}$ e q è messa in evidenza dall'espressione ma è al contrario. In (7), non è veramente $\boldsymbol{p}$ mette in discussione solo alcuni tratti della 
definizione (p) usata che diventa in questo caso un modo possibile di parlare del mondo (rappresentando un punto di vista sul mondo): si tratta sempre della stessa persona (lo scacchista Karpov) ma non ha più tutte le proprietà che gli sono di solito attribuite, per questo si può dire che non è il vero Karpov. Nei due esempi seguenti, il contesto si svolge secondo lo stesso principio.

(8) Eppure il colore, il sentimento del colore, non è davvero un complemento secondario, ma è anzi un elemento fondamentale della volta Sistina. A pari diritto del disegno, anzi da esso indissolubile è un fattore primario di quella univoca intonazione pittorica che circola in tutto l' affresco e unisce storie bibliche, Profeti e Sibille, nudi e Antenati di Cristo in un solo e costante afflato interiore /.../. (La Repubblica)

(9) Anche il segretario del Pci Achille Occhetto, parlando ieri a Torino e a Pesaro sulla festa della Liberazione, ha chiamato in causa la Dc . Celebriamo il 25 aprile ha detto quando una parte del territorio italiano non è veramente libera perché in essa spadroneggiano contropoteri criminali, e mentre la Dc cerca di spostare a destra l'asse politico del paese. (id.)

In (8), la definizione un complemento secondario ( $\mathrm{p}$ ) negata da non è davvero è opposta ad un'altra definizione un elemento fondamentale che sostituisce $\mathbf{p}$ : in questo modo la categoria proposta per parlare del mondo viene sostituita con un'altra. In (9), nel contesto posteriore viene spiegato per quali ragioni la definizione scelta (libera) non è completamente adeguata anche se accettata da tutti: un punto di vista sul mondo può avere diverse interpretazioni.

Le differenze semantiche tra davvero e veramente possono spiegare anche le differenze di carattere stilistico che esistono tra queste due parole ${ }^{5}$ : "Veramente étant focalisé sur les problèmes d'interprétation de la séquence correspondant à sa portée, est plus souvent employé dans le discours informel où l'interlocuteur actif est présent, plusieurs points de vue sont alors mis en jeu. Davvero centré sur le problème de l'existence ou non de $\boldsymbol{p}$ est plus caractéristique du discours formel (les sermons, les programmes télévisés) adressé à un interlocuteur qui a la position passive, car il ne peut pas intervenir." (Khachaturyan, 2009a)

\section{[2.2] Le particelle (classe 2)}

Tradizionalmente, le grammatiche e le descrizioni linguistiche universali distinguono tra le lingue ricche di particelle (p.es. il russo, il tedesco, il greco) e povere di particelle (p.es. le lingue romanze). Tuttavia, accettando la definizione proposta nell'ambito del nostro metodo un gruppo di unità in diverse lingue può essere definito come particelle.

[5] Basti controllare il corpus di C-Oral-Rom per notare la prevalenza della sequenza veramente $\mathrm{p}$ nel discorso informale e l'uso più frequente di davvero $\mathrm{p}$ nel discorso formale. 
"Une particule énonciative signifient que la séquence p correspondant à sa portée est introduite compte tenu de l'actualisation d'une séquence $\boldsymbol{p}$ ' qui est, à des degrés divers, en concurrence avec p. L'altérité $\boldsymbol{p}$ / p' est souvent un enjeu sur le plan intersubjectif." (Paillard \& Vu Thi, in stampa)

Bisogna notare che spesso fanno parte del gruppo di particelle gli elementi tradizionalmente definiti "congiunzione testuale" (p.es. d'altronde, pure, altrimen$t i$, ecc.). A nostro avviso, comunque, non si tratta di una semplice connessione (a differenza delle congiunzioni), ma di un conferimento alla portata $\mathbf{p}$ di uno status discorsivo particolare. Nel caso delle particelle questo status viene definito rispetto ad un altro elemento del contesto. Non è più il rapporto tra $\mathbf{p}$ e lo stato delle cose $\mathbf{Z}$ che viene messo in discussione (come nella classe 1), ma è il rapporto tra $\mathbf{p}$ e p'.

Così, lo status discorsivo della portata $\mathbf{p}$ viene stabilito tenendo conto di un altro elemento p' (in concorrenza con $\mathbf{p}$ ) che può essere presente (come in ex.10, 11) o meno (12) nel contesto precedente.

(10) Questo è un grave sacrificio che ci viene imposto. D'altronde bisogna accettare le regole del gioco democratico. (La Repubblica)

Nell'esempio (10), l'elemento $\mathbf{p}$ ' contiene la visione critica di quello che è stato fatto (Z): un sacrificio - imposto. L'elemento $\mathbf{p}$ (la portata di d'altronde) propone un'altra visione dello stesso evento: non è più un sacrificio, ma sono le regole del gioco, quindi non è più un'azione imposta, ma sono le regole che bisogna accettare. Così nella sequenza discorsiva due visioni opposte di quello che è successo sono presenti: dalla visione critica p' che è in disaccordo con quello che è stato fatto si passa alla visione $\mathbf{p}$ - comprensiva.

Come vediamo, nei contesti con d'altronde $p$ di solito è presente un'altro elemento $\mathbf{p}$ ', con il quale d'altronde stabilisce una certa relazione specificando allo stesso tempo lo status di p (la sua portata) rispetto a p': nello svolgimento del discorso $\mathbf{p}$ segue $\mathbf{p}$ ', però il contenuto di $\mathbf{p}$ è in opposizione al contenuto di p' (si può quasi dire che $\mathbf{p}$ annulla $\mathbf{p}$ '), d'altronde ristabilisce l'equilibrio segnalando che $\mathbf{p}$ è una visione diversa della situazione (10). Lo si può osservare anche nell'esempio (11):

(11) Ormai dobbiamo andarcene. D'altronde abbiamo aspettato abbastanza. (es. dal Sabatini \& Coletti)

Nella prima frase dell'esempio (11), la partenza è presentata come un evento perentorio che non dipende dalla volontà del parlante (ormai dobbiamo). D'altronde introduce un'altra ragione della partenza: è il seguito naturale della lunga attesa (abbiamo aspettato abbastanza). La particella pur attualizza altri membri dello stesso ordine: in (12), altri elementi possono far parte della lista 'essere onesto in' tra 
i quali anche $\mathbf{p}$ (nell'amore).

(12) Perché Jago è onesto. Onesto pur nell' amore. Vuol far tornare i conti del dare e dell' avere erotico. (La Repubblica)

\section{[2.3] I modalizzatori (classe 3)}

Attualmente, il gruppo di modalizzatori è descritto meno degli altri. Per il momento è stata proposta la definizione seguente:

"Les MD modalisateurs sont des MD qui marquent un effet de "brouillage " concernant la sélection de p pour exprimer "ce qui est le cas " (Z). Cet effet de brouillage peut être lié aux mots constituant l'énoncé: d'une certaine façon les mots utilisés ne sont qu'indirectement pertinents pour exprimer ce qui est le cas. Il peut aussi marquer le fait que le sujet n'a pas une perception nette de ce qui est le cas. Ou, enfin, marquer que le rapport de p à $Z$ comporte une part d'indétermination." (Paillard \& Vu Thi, in stampa)

Molti elementi di questo gruppo sono tradizionalmente definiti come "indicatori di riduzione della precisione" (Bazzanella, 1995: 238). Nella lingua parlata diventano spesso tic verbali (o riempitivi) che si caratterizzano di solito dalla desemantizzazione parziale o completa. Secondo noi, però, ogni elemento conserva una certa semantica. Ciò che spiega l'uso nel contesto specifico tipico di ogni elemento.

(13) ... aveva impiegato intere pagine del Popolo d' Italia a denigrare il padre della sua interlocutrice, e poi, giunto al potere, gli aveva praticamente confiscato La Stampa. Ma ora a Luciana Frassati, pur nel dissenso di fondo, il Duce appariva nè crudele, nè spietato (...) (La Repubblica)

(14) l'investimento risale a / millenovecen // duemila ...duemila // - duemila // quindi praticamente / lei s'è beccato tutto il periodo / diciamo / di massimo splendore dei mercati finanziari (C-Oral-Rom)

(15) La lunga serie comincia nel 1961 ma la vicenda clou risale al 1972 . A quell' epoca Argirò era un play boy piuttosto quotato in città , uno che si era creato un giro discreto e cercava di sfruttarlo al meglio per campare alla sua maniera. (La Repubblica)

Le condizioni d'uso di questi elementi restano ancora da definire in modo più approfondito. Sarà fondamentale in questo studio il ruolo dei corpora della lingua parlata grazie ai quali si potrà realizzare una dettagliata analisi prosodica.

È interessante osservare che, a differenza delle altre classi di SDi dove spesso la forma dei SDi coincide in diverse lingue ${ }^{6}$, questi elementi sono caratterizzati

[6] Possiamo osservare che i SDi della classe 1 hanno di solito la forma degli avverbi, i SDi della classe 2 sono spesso definiti come congiunzioni, mentre i segnali del dire (classe 4) sono formati dal verbo (in particolare, dal verbo dire). 
da un'origine molto diversa. Cfr. p.es. in francese: quoi (pronome interrogativo e relativo), in russo: kak by ( $k a k$ - avverbio 'come' + by - particella postverbale usata per formare il condizionale) - in inglese: like (aggettivo).

(16) et moi ce que je fais aussi c' est par exemple je vois des points de grammaire / puis je passe à autre chose / je poi/ je vois un autre point de grammaire puis je rev/ je repasse au point de grammaire qu' on a vu euh tu vois il y a trois mois quoi (corpus d'A. Dister)

(17) - What was your reaction to that? - I mostly just didn't pay attention to it because my family's strong and it doesn't matter what the - like, what tabloids say or anything like that. (web)

\section{[2.4] I segnali del dire (classe 4)}

Questa classe di SDi è caratterizzata da parole della stessa origine: sono, in primo luogo, gli elementi formati dai verba dicendi (p.es. dire, to say - to speak, skazat' govorit' (in russo)). Tra i segnali del dire in italiano possiamo citare gli elementi seguenti: diciamo, per così dire, a dir il vero, vuol dire, dico, ecc. Tuttavia, come vedremo successivamente, non solo i SDi formati dal verbo dire fanno parte di questo gruppo. E'stata proposta la definizione seguente: "Les mots du dire signifient que les mots utilisés par le sujet ressortissent un 'vouloir dire' qui n'est pas le sien. Ils correspondent à une forme de désengagement du sujet et actualisent une distance entre un "vouloir dire " (d'un sujet, des mots, du monde) et l'énoncé." (Paillard \& Vu Thi, in stampa)

I SDi del dire mettono in evidenza il rapporto fra tre componenti della comunicazione ciascuna con il suo vouloir dire (1. interlocutori: So/S; 2 . il mondo che è da dire; 3. le parole (o il detto - le dit)) e segnalano una certa distanza tra il vouloir dire e quello che viene detto ${ }^{7}$.

La semantica dei SDi di questa classe (a differenza delle altre) è più legata alla presenza del locutore - portatore di un certo vouloir dire - che cerca di trovare le parole per trasmettere la sua visione del mondo all'interlocutore. L'interlocutore, a sua volta, le può interpretare a modo suo. Il carattere di questa distanza tra i tre vouloirs dire può essere di vari tipi a seconda del SD che viene usato e quindi può avere delle conseguenze diverse per lo sviluppo del contesto.

Confrontiamo due SDi formati dal verbo dire: diciamo e per così dire. Entrambi sono di solito definiti come meccanismi di modulazione: "possono indicare la mancanza di precisione nella formulazione del contenuto proposizionale e la non completa adeguatezza dell'espressione usata" (Bazzanella, 1995: 238). Vediamo, nell'ambito del nostro approccio, quale status discorsivo viene attribuito da questi elementi alla loro portata $\mathbf{p}$.

[7] Per una descrizione più dettagliata dei problemi di comunicazione v. Culioli, 2002, Culioli \& Normand, 2005. 
(18) Proposte di assoluzione non ce ne saranno molte, ma - ha anticipato il rappresentante dell' accusa - non mancheranno richieste di condanna a pene miti per quegli imputati, diciamo di serie $\mathrm{C}$, che hanno finito per aderire all' organizzazione per delinquere di don Raffaele Cutolo per paura, alcuni addirittura lasciandosi affiliare in carcere per non rimanere tagliati fuori, per tutelare la propria incolumità, perché costretti a scegliere: o con quelli della Nuova camorra organizzata o contro di loro. (La Repubblica)

(19) Mi sembra logico che i partiti vincitori di elezioni nelle quali hanno impegnato grandi energie alla fine tendano, per così dire, a riscuotere. Da quando esistono le elezioni in Italia è sempre andata così. Sarebbe successa la stessa cosa se a vincere fosse stato Fumagalli. (Il Corriere della Sera)

(20) Si discute se nel match non tutto sia stato trasparente. L'Ibm non ha infatti permesso a Kasparov di esaminare i tabulati di Deep Blue e non intende pubblicarli integralmente: lo ha programmato inglobando nei suoi circuiti tutti i precedenti match del campione, che invece ha giocato per così dire al buio. (id.)

Diciamo mette in evidenza la collaborazione di S0 con S1 nell'interpretazione di $\mathbf{p}$ ( $\mathbf{p}$ corrisponde a un modo possibile di dire $\mathbf{Z}$ condiviso con S1): in (18) per parlare degli imputati meno importanti del processo antimafia viene usato il termine calcistico di serie $C$ che generalmente è usato per indicare squadre meno titolate.

Nel caso di per così dire, invece, è il vouloir dire delle parole (p) stesse che è messo in primo piano, mentre So prende le sue distanze rispetto a quello che dice: in (19), la parola riscuotere usata senza oggetto diretto può essere interpretata in modi diversi (riscuotere soldi o riscuotere successo). In (20), l'espressione giocare al buio che di solito viene associata al gioco del poker va interpretata diversamente nel contesto: 'senza informazione'. Possiamo notare che in questi SDi la forma stessa del SD influisce notevolmente sulla semantica. (Khachaturyan, 2006, 2010b)

\section{[3] APPLICAZIONI PRATICHE}

Le difficoltà legate alla descrizione e l'uso dei SDi possono essere descritte tramite domande molto pratiche: come tradurre i SDi? come impararli in una lingua straniera? come spiegare la differenza tra SDi molto simili? Il metodo che noi usiamo può aiutare a dare alcune risposte.

\section{[3.1] Sinonimia e polifunzionalità dei segnali discorsivi}

I SDi sono tradizionalmente considerati come elementi polifunzionali con una sinonimia molto ricca. Questo fatto rende l'acquisizione dei SDi ancora più difficile. La classificazione che abbiamo proposto permette di strutturare la classe di SDi 
basandosi sulle proprietà formali di carattere sintattico e semantico. Così, la descrizione di ogni SD attraverso la sua intrinseca identità semantica e le variazioni nel contesto, da un lato, permette di unificare le proprietà dei SDi polifunzionali, dall'altro lato, aiuta a distinguere tra SDi simili con le stesse funzioni pragamtiche.

Come abbiamo visto, ogni SD ha la sua identità semantica che rimane invariabile in tutti i contesti, allo stesso tempo i SDi appartenenti alla stessa classe hanno le proprietà semantiche in comune. Inoltre, i SDi ai quali tradizionalmente sono associate le stesse funzioni pragmatiche, nel caso della descrizione semanticoformale spesso appartengono a classi diverse. Analizziamo alcuni esempi per illustrare ciò che è stato detto.

a) Funzione di focalizzazione: davvero e dico.

Confrontiamo i SDi davvero e dico che sono di solito definiti come segnali di focalizzazione (Bazzanella, 1995: 238, 247). Analizziamo entrambe le parole usate in un contesto simile: in (21-24) la portata $\mathbf{p}$ contiene un quantificatore tutto. È interessante esaminare come cambia lo status della portata e tutto il contesto a seconda della parola che lo introduce.

(21) Un giovane volontario canadese, Ted Allan, s'affacciò una sera nella hall dell'albergo "Florida", e si vide passare sotto gli occhi Pablo Neruda e Dos Passos, Malraux e Arthur Koestler, Hemingway ed Ehrenburg, SaintExupery, Lilian Hellman e Rafael Alberti. Sbalordito, scrisse la sera stessa ad un amico: "Ci sono tutti, ti dico tutti, salvo Shakespeare". (La Repubblica)

(22) io / quando si parla di queste cose / molto probabilmente per carattere mio / sono sempre sconcertato / perché / tutti i giorni / dico / tutti i giorni / sia alla televisione / sia su quotidiani / e roba del genere / siamo martellati dal / tutte quelle notizie / che producono / o più che producono / che danno / noia (C-Oral-Rom)

Dico come tutti i SDi del dire mette in evidenza il legame fra le tre componenti della communicazione e i loro vouloirs dire: le parole usate dal locutore per parlare del mondo. Così, dico è usato per sottolineare che $\mathbf{p}$ è un'espressione o una parola scelta apposta dal locutore: il vouloir dire di $\mathbf{p}$ è perfettamente adatto per parlare del mondo. ${ }^{8}$

(23) Davvero tutti i tatuaggi possono essere scaricati gratuitamente? (web)

(24) Paypal rimborsa davvero tutti (o quasi). (web)

[8] Questa autorità marcata crea spesso il carattere polemico di tutto il contesto dove viene usata la forma dico. Cfr: ma ma dico io e' una cosa pazzesca che al giorno d' oggi ci sia della gente frivola fa un questionario per dire per dire eh il vangelo si attacchi al cazzo eh o qualche altra cosa eh sa mia figlia prima di sposarsi vuol convivere ahah che brutta cosa (LIP) 
Davvero invece, come tutti i SDi di adeguatezza, mette in discussione l'adeguatezza della parola $\mathbf{p}$ (che è dell'ordine della verità assoluta) per parlare del mondo. È tanto più marcato quando $\mathbf{p}$ ha la forma di un quantificatore come in $(23,24)$.

Così, la funzione pragmatica di focalizzazione può essere descritta diversamente secondo l'approccio che proponiamo: a seconda del SD usato, la focalizzazione può corrispondere alla marcata (non) adeguatezza tra $\mathbf{p}$ e $\mathbf{Z}$ basata su diversi criteri (verità, realtà, ecc.), come nel caso dei SDi adeguatezza e SDi punto di vista $^{9}$, oppure alla marcata distanza tra $\mathbf{p}$ e $\mathbf{Z}$ dove $\mathbf{p}$ con tutte le interpretazioni possibili è al centro (segnali del dire).

b) Funzione di mitigazione: diciamo e praticamente.

Tra i SDi che hanno la funzione di mitigazione si trovano sia i modalizzatori che i SDi del dire. Tuttavia, se i modalizzatori segnalano solamente una certa inadeguatezza tra $\mathbf{p}$ e $\mathbf{Z}$ basata sulla non pertinenza delle parole, nel caso dei SDi del dire (a causa della forma verbale e della semantica del verbo stesso) gli interlocutori sono direttamente coinvolti nell'impresa che consiste nel dire $\mathbf{Z}$ attraverso p.

Così, per esempio, in base alla descrizione dei SDi del dire che abbiamo proposto, l'individualità semantica di diciamo può essere spiegata in modo seguente. Diciamo introduce una denominazione segnalando che le parole $\mathbf{p}$ che corrispondono alla sua portata non sono completamente adeguate per parlare dello stato delle cose $\mathbf{Z}$, è un dire soggettivo e la collaborazione di S1 è necessaria per stabilire il rapporto tra $\mathbf{p}$ e $\mathbf{Z}$.

Questa definizione rimane invariabile in tutti i contesti d'uso di diciamo. Allo stesso tempo può essere reinterpretata a seconda del contesto: le caratteristiche 'non completamente adeguato' e 'la collaborazione di S1' possono essere precisate. In base a queste precisazioni possiamo distinguere diversi tipi di diciamo (Khachaturyan, 2010). Analizziamo due esempi (25) e (26). In (25), la funzione di diciamo è tradizionalmente definita come attenuante (p.es. Hölker 2003: 138).

... infatti li comprendi li comprendiamo fra quelle popolazioni che vivono anche adesso allo stato - i nomadi? - diciamo quasi primitivo sì ecco vorrei invece fare un'altra domanda (LIP, es. presente anche in Hölker, 2003: 138)

In (25), diciamo porta sul sintagma quasi primitivo dove quasi attenua la denominazione primitivo. L'espressione stato primitivo può essere considerata un'espressione fissa (spesso usata per parlare di alcuni popoli) e, quindi, non appartenente al parlante. Essendo un'espressione fissa è possibile che $\mathbf{p}$ non sia completamente adeguato per parlare della situazione concreta in questione. La collaborazione di

[9] Questo gruppo di SDi in italiano deve ancora essere descritto, per il francese v. p.es. Franckel \& Paillard, 2008. 
S1 serve per accettare questo status di $\mathbf{p}: \mathbf{p}$ corrisponde a un dire condiviso con l'interlocutore.

Anche in (26), la portata di diciamo è un'espressione fissa, però deve essere reinterpretata nel contesto. Non è più una generalizzazione come in (25), ma l'individualizzazione della situazione descritta.

(26) - ... Roscio aveva scoperto la tresca della moglie col cugino: li aveva sorpresi insieme. /.../ - Ma dico: Roscio, trovando la moglie diciamo in dolce colloquio con quell'altro, che ha fatto? (L. Sciascia, A ciascuno il suo, XVIII)

In (26), la situazione (lo stato delle cose) è già descritta prima dal soggetto parlante con il termine abbastanza forte - la tresca. Il locutore usa un'altra espressione in dolce colloquio che può essere considerata un'espressione attenuante visto che si sa già di che cosa si tratta. Così p diventa una denominazione alternativa di quello che è successo. A priori $\mathbf{p}$ è un'espressione poco adatta per parlare dell'adulterio. È diciamo che aiuta a stabilire l'accordo reciproco tra gli interlocutori segnalando $\mathbf{p}$ come una possibilità di parlare del mondo, non è un dire condiviso (come in (25)), ma un dire figurato.

Così, se nel caso di diciamo è importante prendere in considerazione diverse interpretazioni possibili che può avere $\mathbf{p}$ (per questo diciamo accompagna spesso l'uso figurato e il gioco di parole), gli esempi con praticamente sono focalizzati sull'inadeguatezza delle parole, ma non perché diverse interpretazioni vanno previste, ma perché a priori il legame tra p e Z è "disturbato"10 (cfr. (13 e 14)). Questa differenza può spiegare, per esempio, l'alta frequenza di praticamente usato con gli elementi che a priori possono avere una sola interpretazione (come, per esempio, gli elementi deittici oppure i quantificatori).

(27) Il grande Borg, che si è ritirato a ventisette anni, dopo aver vinto praticamente tutto, a chi gli chiedeva a cosa pensasse di solito prima della partita, rispondeva: a quante ore dovrò stare in campo per aver ragione del mio avversario. (La Repubblica)

\section{[3.2] Analisi contrastiva}

È ben noto quanto sia difficile trovare un SD equivalente in un'altra lingua. Questo fatto spiega anche le difficolta nell'acquisizione di L2 (p.es. Lamiroy, 1994). Le parole della stessa forma spesso hanno funzioni completamente diverse, mentre le parole che sembrano diverse dal punto di vista formale possono avere funzioni simili (p.es. Rossari, 1994). Le difficoltà sono ancora più notevoli quando si tratta di due lingue della stessa origine (come, per esempio, l'italiano e il france-

[10] Per questo nella definizione dei modalizzatori si parla dell'effetto di brouillage. 
se). In questo caso la presenza dei criteri formali (di carattere morfosintattico e semantico) può dare degli strumenti necessari per un'analisi contrastiva.

a) Diciamo - disons

Confrontiamo l'uso di diciamo e disons nel contesto di riformulazione: q diciamo / disons $\mathbf{p}$ - dove sia $\mathbf{q}$ che $\mathbf{p}$ sono usati per denominare lo stato delle cose $\mathbf{Z}$.

(28) Se Alain Juppé dovesse dimettersi, voi preferireste un uomo come Philippe Séguin, neogollista, diciamo, di sinistra? Il presidente dell'Assemblea, nemico del trattato di Maastricht, è andato a dialogare coi ferrovieri e a distribuire pacche sulle loro spalle. (Il Corriere della Sera)

(29) Stéphane Israël. A la différence de la génération de 1968, la nôtre rassemble une multiplicité de micro-générations : génération Mitterrand, bof génération, génération morale. Du point de vue politique, je discerne trois événements fondateurs. D'abord, évidemment, la gauche au pouvoir. Ensuite, la fin du communisme, disons la chute du Mur de Berlin. Enfin, la crise sociale. (Le Monde)

Nel caso di diciamo, vengono prese in considerazione diverse interpretazioni possibili della parola (p) usata: in (28), l'espressione di sinistra viene reinterpretata nell'ambito della definizione precedente neogollista (quindi a priori di destra). Si tratta di una denominazione particolare dello stato delle cose. Nel caso di disons, viene messo in evidenza un altro problema - l'insufficienza delle parole (lo illustra bene (30)) e quindi la necessità di trovare una denominazione di compromesso oppure usare diversi modi per dire Z Z. In (29), la denominazione $\mathbf{p}$ non ha niente di particolare: la fine del comunismo a priori può essere associata alla caduta del muro di Berlino. Si tratta solo di un altro modo di dire il mondo.

(30) On lui a offert, pour son anniversaire, de belles places, à Covent Garden. Il est assis. Et voici que survient, à la place voisine, une belle personne enfin belle n'est pas le mot... disons l'une des femmes les plus élégantes, les plus glamourous, qu'il ait jamais rencontrées : ils se reconnaissent, elle n'a probablement pas lu ses livres mais a la courtoisie de faire comme si. Bonsoir? Bonsoir. Echange de propos. Rideau. (Le Monde)

Per spiegare queste differenze possiamo rivolgerci alla semantica verbale che contribuisce alla semantica del SD. Nonostante il verbo dire in italiano e francese abbia la stessa origine ${ }^{11}$ e molti usi simili ${ }^{12}$, è possibile notare alcune differenze che in-

[11] Secondo il dizionario etimologico, il verbo dire risale alla radice indo-europea *deik-, * dik- « mostrare » presente anche nel verbo tedesco zeigen ('mostrare') e nel verbo greco $\delta \varepsilon i \chi \nu \omega ~(' m o s t r a r e ')$.

[12] È da segnalare in questo caso la sinonimia con il verbo 'pensare' (a differenza del verbum dicendi russo (skazat'), per esempio). Cfr.: Nelle espressioni seguenti dire può essere sostituito con il verbo pensare: $E$ dire che - et dire que, Chi l'avrebbe detto! - qui l'eût dit! Si direbbe - on dirait. 
fluiscono sulla semantica dei SDi del dire (Khachaturyan, 2010b). Riportiamone solo alcune.

- Frase interrogativa

Una frase interrogativa con il verbo italiano e con il verbo francese sarà interpretata in modo diverso.

(31) Stasera mangiamo il risotto, che dici? (es.reale)

(32) Bilardo, ma che dici? L'Argentina senza Maradona sarebbe niente! (web)

(33) quoi ? hein? qu'est ce que tu dis? je n'entends pas. (web)

La frase interrogativa con il verbo italiano è sempre rivolta al contenuto di quello che è stato detto, è usata per esprimere l'accordo (in (31), può essere riformulata come 'sei d'accordo?' o il disaccordo (p.es. in (32)). La frase con il verbo francese (33) chiede della forma e corrisponde a una richiesta di ripetere ciò che è stato detto.

- Negazione

Le costruzioni equivalenti dal punto di vista formale, costituite con elementi simili (senza/sans + dire/dire + NEG) sono usate in contesti diversi.

(34) E ho coinvolto un altro dei miei miti, Hugo Pratt. Fa un commissario straniero che arriva, guarda, capisce tutto e se ne va senza dire niente. Ha capito che i delitti non sono roba sua, è roba da psicanalista bravo. (Il Corriere della Sera)

(35) Una scuderia decide di montare un motore di 3500, 4000, 5000 cc anziché di $3000 \mathrm{cc}$ come da regolamento e così facendo vince alla grande senza dire niente al pilota. (id.)

(36) Era ... strano. Dopo l' orrore, mi riempiva di gentilezze. Entrando alla dacia avevo guardato un albero di limoni. Solo uno sguardo, senza dire niente: e subito mi fa trovare una cesta di limoni in tavola. L' auto che mi riporta a Mosca è piena di rose, completamente piena. (id.)

(37) [la femme ne sait pas cuisiner] Quand la femme de ménage est en vacances, l'Homme passe, sans rien dire, chez le traiteur chinois et rapporte de délicieux plats de poulet aux champignons noirs, des crevettes au gingembre, du canard laqué, du riz cantonnais, des lychees /.../ (Frantext, N. de Buron)

(38) Vous avez aussi appris à : ...ne rien dire quand il grogne à voix haute au théâtre : "Quelle pièce de merde! Et l'actrice, une vieille peau... » alors qu'il s'agit d'une de vos amies qui vous a gentiment invités. (id) 
Il verbo italiano con la negazione è spesso usato nella situazione di comunicazione dove gli eventuali interlocutori si aspettano che il locutore parli (mettendo il proprio vouloir dire in parole per trasmetterlo a S1): in (34), l'eventuale locutore sa qualcosa, in (35) fa qualcosa ma non lo dice al potenziale interlocutore, non lo avverte, in (36) lo sguardo sostituisce le parole.

Il verbo francese con la negazione non prevede la situazione di comunicazione: è usato quando l'eventuale locutore non reagisce, non esprime le sue emozioni verso quello che è stato detto (38) o fatto (37) e che dovrebbe suscitare la sua reazione. Così, nel caso del verbo francese, la focalizzazione sulle parole viste come forma che permette di 'mettere in scena' (usando il termine di D.Paillard, 2009) o esprimere il proprio vouloir dire è presente anche nei segnali del dire in francese. Nel caso del verbo italiano, è la presenza di $\mathrm{S} 1$ che è presa in considerazione, la divergenza tra S0 e S1 è più marcata: le parole usate da S0 danno accesso al suo proprio vouloir dire che è diverso da quello di S1. I segnali del dire in italiano si caratterizzano da una forte alterità. Queste differenze possono anche spiegare la presenza degli elementi di un'altra origine tra i segnali del dire francesi.

b) Tu vois.

$\mathrm{Tu}$ vois è molto frequente nel francese parlato. ${ }^{13}$ In italiano tra gli elementi più diffusi del parlato spontaneo sono i SDi del dire diciamo e dico ${ }^{14}$. Il SD tu vois è definito come "appel à la prise en considération de ce qui se dit" (Morel \& DanonBoileau, 1998: 96). Ci siamo proposti di capire quali sono le proprietà individuali di tu vois che lo distinguono dagli altri elementi con la stessa funzione (e.g. écoute) provocando la sua maggiore frequenza nel parlato spontaneo e, secondo la nostra ipotesi, avvicinandolo ai SDi del dire (come p.es. dico e diciamo in italiano). Per rispondere a queste domande abbiamo analizzato i contesti d'uso di tu vois e ne abbiamo individuati tre tipi a seconda del carattere di p:

- p è una denominazione particolare di Z (39),

- $\mathbf{p}$ è un termine generico (40),

- $\mathbf{p}$ è una delle possibili denominazioni di Z (41). ... mais c' est dingue quoi aussi et c' est marrant parce que // je sais pas si je te l' avais dit je sais plus à qui j' ai dit ça mais / je c' est un truc de euh / je trouve que c' est vraiment un truc de fille tu vois de de de prendre la tête tout le temps et on va faire ça et on va faire ç/ enfin moi je me reconnais vraiment dans ce comportement-là tu vois // (corpus d'A. Dister)

[13] Cfr. l'analisi di S.Schneider (2007) secondo la quale il 19,8\% delle incise nel francese parlato corrisponde a tu vois.

[14] Secondo la stessa analisi di S.Schneider il loro uso corrisponde al $41 \%$ e al 7,5\% rispettivamente. 
In (39), l'espressione un truc de fille (la portata $\mathbf{p}$ di tu vois) corrisponde a una denominazione particolare/individuale dello stato delle cose $\mathbf{Z}$, che viene spiegata nel contesto posteriore (sottolineato).

(40) blaND1 j' ai déjà -| un peu parlé de mes vacances d' ailleurs blaAT1 ah / ouais ouais tu as bien fait / et quoi? blaND1 ben elle saura pas me dire avant juin donc euh j' étais / mais euh blaAT1 bah oui / mais ils savent pas prévoir hein dans ce truc-là / |- ils savent

<blaND1> m -| pas prévoir moi je trouve ça dingue quoi tu vois / je suis désolée en septembre tu es tu tu es censé euh savoir euh // enfin tu as pas l' impression toi ? je sais bien que c' est des sites différents mais qu' on demande euh / (sonnerie de téléphone) (id.)

In (40), $\mathbf{p}$ è una definizione molto vaga che può avere diverse interpretazioni a seconda del contesto (secondo il TLF: "dingue" può significare "fou, bizarre, extraordinaire"). Così p può essere sostituito con un'altra parola o espressione che può essere usata per dire $\mathbf{Z}$. Dicendo $\mathbf{p}$ tu vois il locutore lascia la possibilità di introdurre una denominazione alternativa. Allo stesso tempo per dare l'interpretazione giusta a quello che è stato detto serve la collaborazione dell'interlocutore. Bisogna notare che la presenza di tu vois suscita spesso la reazione dell'interlocutore che conferma la "ricezione" di p, come nell'esempio seguente.

(41) MB N 2: A : / il y a des tours operators / qui proposent des voyages / où \& avec \&euh\& une fille par soir /

$\mathrm{B}: \mathrm{oh}$

A : tu vois //

B : oh putain

A : par exemple // mais c'est compris dans le prix du voyage / tu vois // (C-Oral-Rom)

In tutti questi esempi è possibile individuare alcuni tratti comuni che possono essere formulati in modo seguente. Tu vois conferisce alla sua portata lo status di un elemento che corrisponde a un modo personale (appartenente a S0) di parlare di $\mathbf{Z}$, essendo un modo personale può essere sostituito con un altro elemento (p'), anche $\mathbf{p}$ ' è adeguato per parlare di $\mathbf{Z}$. Allo stesso tempo il fatto che $\mathbf{p}$ sia un modo personale di dire $\mathbf{Z}$ richiede una certa collaborazione dell'interlocutore che deve accettare $\mathbf{p}$ come una delle possibilità di dire $\mathbf{Z}$.

Così, come tutti i SDi del dire, tu vois segnala la distanza tra p (un dire personale) e lo stato delle cose $\mathbf{Z}$, detto attraverso $\mathbf{p}$, tenendo conto della presenza di S1. In questo modo, tu vois unisce le proprietà di diciamo e di dico: diciamo segnala che $\mathbf{p}$ non è completamente adeguato per dire $\mathbf{Z}$ (cfr. (40) con (25), (39) con (26), 
per accettarlo serve la collaborazione di S1. Dico mette in evidenza l'autorità di So (S0 come autore di $\mathbf{p}$ ) e $\mathbf{p}$ come modo personale di dire $\mathbf{Z}^{15}$.

\section{[4] CONCLUSIONI}

Come abbiamo visto, l'approccio presentato in questo articolo permette di dare una presentazione sistematica delle unità linguistiche che, pur essendo molto frequenti e indispensabili nella lingua, sono di solito poco (se non per niente) descritte nelle grammatiche e nei manuali di lingua straniera. La classificazione proposta essendo basata sui criteri formali (di carattere morfosintattico e semantico ${ }^{16}$ ) permette di completare questa mancanza.

[15] Bisogna notare che la forma vedi in italiano, pur avendo l'uso discorsivo, ha il ruolo completamente diverso nel contesto. Anche in questo caso la semantica verbale può fornirci una spiegazione ma si tratta del lavoro che resta ancora da fare.

[16] A questi criteri formali vanno aggiunte anche le caratteristiche prosodiche. È un argomento interessante ma per il momento poco studiato (p.es. Bonnot \& Kodzassov, 1998). 
RIF ERIMENTI B IB LIOGRAFICI

Andersen, G. 2000. The role of the pragmatic marker like in utterance interpretation. Pragmatic markers and propositional attitude .

Authier-Revuz, J. 1995. Ces mots qui ne vont pas de soi: boucles réflexives et non-coïncidences du dire, vol. 1 e 2. Paris: Larousse.

Bazzanella, C. 1995. I segnali discorsivi. In L. Renzi, G. Salvi \& A. Cardinaletti (a cura di), Grande grammatica italiana di consultazione, vol. III, 225-257. Bologna: Il Mulino.

Bazzanella, C. 2006. Discourse markers in Italian: Towards a "compositional" meaning. In K. Fisher (a cura di), Approaches to discourse markers, vol. Studies in Pragmatics 1., 449-464. Amsterdam: Elsevier.

Bonnot, C. \& Kodzassov S. 1998. La variation sémantique des mots du discours et son influence sur la linéarisation et l'intonation de l'énoncé (en russe). In K. Kisseleva \& D. Paillard (a cura di), Diskursivnye slova russkogo jazyka: opyt kontekstno-semantičeskogo analiza. (Les Mots du discours du russe : essaie de description sémantico-contextuelle), 382-443. Moskva: Metatekst.

Culioli, A. 1990. Pour une linguistique de l'énonciation: opérations et représentations, vol. 1. Paris: Editions Ophrys.

Culioli, A. 2009. Variations sur la linguistique. Entretiens avec Frédéric Fau. Paris: Klincksieck.

Culioli, A. \& C. Normand. 2005. Onze rencontres sur le langage et les langues. Paris: Editions Ophrys.

Fischer, K. 2006. Approaches to discourse markers, vol. Studies in Pragmatics 1. Amsterdam: Elsevier.

Franckel, J.J. \& D. Paillard. 2008. Mots du discours: Adéquation et Point de vue. L'exemple de 'réellement', 'en réalité', 'en effet', 'effectivement'. In H. T. Valentim \& B. Moreira (a cura di), Estudos linguísticos / linguistic studies 2, 255-274. Lisboa: Edições Colibri / CLUNL.

Fraser, B. 1990. An approach to discourse markers. Journal of Pragmatics 14(3). 383-398.

Hölker, K. 2003. It. diciamo als Mitigator. In G. Held (a cura di), Partikeln und höflichkeit, 131-153. Bern: Peter Lang Publishing.

Khaciaturian, E. 2005. Sul segnale discorsivo senti. Studi di grammatica italiana vol. XX. 127-137.

Khatchatourian, E. 2006. Les mots du discours formés à partir des verbes dire / skazat' (en français et en russe). Ph.D. thesis, University Paris 7.

Khatchatourian, E. 2008. Les mots du discours de dire. Analyse contrastive. Revista de Estudos Linguisticos n. 2. 287-315.

Khachaturyan, E. 2009a. Les mots du discours: le lien entre les propriétés sémantico-contextuelles et les propriétés prosodiques. (Analyse des mots 
du discours italiens davvero et veramente). Travaux Linguistiques du CerLiCo Vol. 22. 189-207. Presses universitaires de Rennes.

Khachaturyan, E. 2009b. Segnali discorsivi formati dal verbo dire. Approccio semantico. In Proceedings of the 8th Conference of Scandinavian Italianists, 348-362. Aarhus Universitet: Institut for Sprog, Litteratur og Kultur.

Khachaturyan, E. 2010a. Si tu vois ce que je veux dire par là : l'analyse du verbe dire/dire en français et en italien. Communication presentée au Congrès International de Linguistique et Philologie Romane (CILFR 2010), Valencia, 6-11 septembre, 2010.

Khachaturyan, E. 2010b. The Function of Mitigation in Spoken Language. Analysis of tak skazat' (Russian) and diciamo (Italian). In A. Grønn \& I. Marijanovic (a cura di), OSLa revue. Proceedings of the International Conference "Russian in Contrast", vol. 2, 393-412.

Kisseleva, K. \& D. Paillard (a cura di). 1998. Diskursivnije slova russkogo jazika. (Les Mots du discours du russe : essaie de description sémantico-contextuelle). Moskva: Metatekst.

Lamiroy, B. 2009. Pragmatic connectives and L2 acquisition: The case of French and Dutch. Pragmatics 4(2). 183-201.

Morel, M.A. \& L. Danon-Boileau. 1998. Grammaire de l'intonation. L'exemple du français oral. Paris: Editions Ophrys.

Paillard, D. 1998. Les mots du discours comme mots de la langue I et II. Le Gré des langues 14 \& 16 .

Paillard, D. 2002. Les mots du discours. Identité sémantique et principes de variation. Cahiers Linguistiques de l'INALCO 4. 31-47.

Paillard, D. 2009. Prise en charge, commitment ou scène énonciative. Langue française 162. 109-128.

Paillard, D. \& N. Vu Thi (a cura di). (In corso di stampa). Inventaire raisonné des marqueurs discursifs du français. Université Nationale de Hanoï.

Pons Bordería, S. 2006. A Functional Approach for the Study of Discourse Markers. In K. Fisher (a cura di), Approaches to discourse particles, vol. Studies in Pragmatics 1, 77-100. Elsevier.

Rossari, C. 1994. Les opérations de reformulation: analyse du processus et des marques dans une perspective contrastive français - italien. Berne: Peter Lang.

Schiffrin, D. 1988. Discourse markers. Cambridge: Cambridge University Press.

Schneider, S. 2007. Reduced parenthetical clauses as mitigators: A corpus study of spoken French, Italian and Spanish. John Benjamins Publishing Co.

Weydt, H. 1977. Aspekte der Modalpartikeln. Studien zur Deutschen Abtoenung. Tuebingen: Niemeyer. 
CORPORA USATI

- per la lingua parlata

C-Oral-Rom - Cresti, E. \& Moneglia, M. (éds.), 2005, C-ORAL-ROM. Integrated Reference Corpora for Spoken Romance Languages, John Benjamins, Amsterdam/ Philadelphia.

Corpus d'A. Dister - Dister A. 2007. De la transcription à l'étiquetage morphosyntaxique. Le cas de la banque de données textuelles orales Valibel, Thèse de doctorat, Université catholique de Louvain, Louvain-la-Neuve.

LIP - Corpus del Lessico di frequenza dell'italiano parlato.

- per la lingua scritta

Frantext

Inoltre, i corpora dei giornali disponibili on-line:

Il Corriere della Sera

La Repubblica

Le Monde

CONTATTO DELL'AUTORE

Elizaveta Khachaturyan

University of Oslo

Department of Literature, Area Studies and European Languages

P-box1003, Blindern

N-0315 Oslo

Norway

elizaveta.khachaturyan@ilos.uio.no 